\title{
Innovation oncological rehabilitation: Applicability of the different techniques physiotherapeutic post breast cancer
}

\author{
Izabela Lopes Mendes* \\ Universidade do Vale do Paraíba, Instituto de Pesquisa e Desenvolvimento, Brazil
}

Breast cancer in Brazil, has been presenting great relevance in health care services, mainly in relation to the epidemiological profile of the disease, and gained space with measures of prevention and control, in order to promote modifications. Positive in the context of the Brazilian population [1].

The surgical approach to breast cancer has evolved substantially over the last few decades. Mastectomy remains an important option, however, in early stage of breast cancer, the conservative surgery followed by radiotherapy is a safe alternative, with acceptable rates of local recurrence, overlife and aesthetic results. Both surgical techniques involve dissection of axillary lymph nodes, because axillary lymph nodes are common sites of breast carcinoma metastasis, and axillary status is an important indicator of prognosis through histological examination [2].

Clinical complications arising from the surgical treatment of breast cancer, whatever the modality adopted, associated with axillary dissection, can reach $70 \%$, and include mainly pain, lymphedema, sensory dysfunction and shoulder motor, which It generates considerable impact in the short and long term on the quality of life of women [3].

The survival rates of 5 years after breast cancer have been reported in $89.2 \%$ of cases, with lower rates in low-income countries, however, it is expected that the increase in survivability due to advances in treatment, greater awareness of the population and Early detection. Studies suggest that morbidities after the treatment of Breast cancer may persist in the long term, with $20 \%$ to $32 \%$ of breast cancer survivors reported aggravated in lymphedema, pain and shoulder dysfunction after a year of primary treatment [4].

Rehabilitation has become an integrative part of clinical care after breast cancer, with level 1 of evidence and degree of recommendation a according to the American Society of Clinical Oncology, which emphasizes the importance to health professionals about the Monitoring and management of patients with breast cancer, focusing on health promotion, evaluation and management of physical, psychological, spiritual and social effects of short and long-term women after treatment [5].

Within the traditional treatments recommended for rehabilitation after breast cancer, the detailed clinical evaluation referring to the strength and function of the shoulder joint with the assistance of biomedical instrumentation, as well as the development and applicability of new technologies in the rehabilitation process, are of paramount importance in the recovery of physical-functional and emotional changes, as well as in the self-esteem and body image of women after breast cancer, with consequent reintegration in the social conviviality and Improve the quality of life.
Robotic technology has been gaining prominence in the field of rehabilitation, increasingly used to treat upper limb motor deficits with high intensity training, one of the determinants in motor recovery by producing results Favorable in relation to motor control and increase the ability to carry out daily life activities, restoring the functionality of the upper limbs while performing repetitive tasks [6,7].

Trujillo et al. [8] emphasized the clinical relevance of robotbased systems, which are advantageous due to programmable motion patterns, including functional movements that allow the patient to perform training independently in relation to therapist, and simulate the activities of daily life.

Virtual reality therapy is an innovative tool in the field of physical and cognitive rehabilitation, by minimizing disturbances of body perception, pain frames and improving movement patterns, which provides a Feedback visual, tactile and verbal through the involvement of the individual in therapy, mainly causing distraction in relation to pain and efforts to carry out activities [9].

According to the literature, with virtual reality therapy it becomes possible to perform functional activities in interactive environments, challenging and safe, which favors the learning and motor control. The training strategy involves Cognitive elements, strength training, endurance and balance, with the aim of stimulating the individual in a broader way, which provides improved functionality and acceleration in the rehabilitation process when compared to conventional techniques [10].

In the face of the above, the importance of Clinical Research In relation to the short-and long-term physiotherapeutic intervention of primary treatment due to breast cancer, with an emphasis on the rehabilitation of acute and chronic clinical complications according to each woman's need, including education, restoration of motor functions and preventive measures through new technologies that contribute to adequate and thorough clinical evaluation, besides innovation in the whole rehabilitation process.

\section{References}

1. Ministério Da Saúde (2018) Instituto Nacional de Câncer José Alencar Gomes da Silva (INCA). Estimativa 2018 - Incidência de Câncer no Brasil. Rio de Janeiro: INCA.

2. Cheng H, Clymer JW, Ferko NC, Patel L, Soleas IM, et al. (2016) A systematic review and meta-analysis of harmonic technology compared with conventional techniques in mastectomy and breast-conserving surgery with lymphadenectomy for breast cancer Breast Cancer (Dove Med Press) 8: p. 125-140 [Crossref]

Correspondence to: Izabela Lopes Mendes, Universidade do Vale do Paraíba, Instituto de Pesquisa e Desenvolvimento, São José dos Campos, Brazil, E-mail: izabela_mendes@outlook.com.br

Received: March 10, 2018; Accepted: March 16, 2018; Published: March 19, 2018 
3. Wilson DJ (2017) Exercise for the patient after breast cancer surgery. Semin Oncol Nurs 33: 98-105. [Crossref]

4. Mafu TS, September AV, Shamley D (2018) The potential role of angiogenesis in the development of shoulder pain, shoulder dysfunction, and lymphedema after breast cancer treatment. Cancer Manag Res 10: 81-90. [Crossref]

5. Runowicz CD, Leach CR, Henry NL, Henry KS, Mackey HT, et al. (2016) American Cancer Society/American Society of Clinical Oncology Breast Cancer Survivorship Care Guideline. CA Cancer J Clin 66: 43-73. [Crossref]

6. Longhi M, Merlo A, Prati P, Giacobbi M, Mazzoli D (2016) Instrumental indices for upper limb function assessment in stroke patients: a validation study. $J$ Neuroeng Rehabil 13: 1-11. [Crossref]
7. Pan L, Song A, Duan S, Yu Z (2017) Patient-Centered Robot-Aided Passive Neurorehabilitation Exercise Based on Safety-Motion Decision-Making Mechanism. Biomed Res Int 2017: 1-11. [Crossref]

8. Trujillo P, Mastropietro A, Scano A, Chiavenna A, Mrakic-Sposta S, et al. (2017) Quantitative EEG for Predicting Upper-limb Motor Recovery in Chronic Stroke Robotassisted Rehabilitation. IEEE Trans Neural Syst Rehabil Eng 99: 1-10. [Crossref]

9. Roosink M, Robitaille N, McFadyen BJ, Hébert LJ, Jackson PL, et al. (2015) Realtime modulation of visual feedback on human full-body movements in a virtual mirror: development and proof-of-concept. J Neuroeng Rehabil 12: 2-10. [Crossref]

10. Molina KI, Ricci NA, de Moraes SA, Perracini MR (2014) Virtual reality using games for improving physical functioning in older adults: a systematic review. J Neuroeng Rehabil 11: 156. [Crossref]

Copyright: $(2018$ Mendes IL. This is an open-access article distributed under the terms of the Creative Commons Attribution License, which permits unrestricted use, distribution, and reproduction in any medium, provided the original author and source are credited. 